\title{
Entanglement entropy of nonlocal theories in AdS spacetimes
}

\author{
Gaurav Narain ${ }^{1, *}$ and Nirmalya Kajuri, ${ }^{2, \dagger}$ \\ ${ }^{1}$ Department of Space Science, Beihang University, Beijing 100191, China \\ ${ }^{2}$ Chennai Mathematical Institute, Siruseri, Kelambakkam 603103, India
}

(Received 30 December 2018; published 24 June 2019)

\begin{abstract}
We investigate the effect of nonlocality on entanglement entropy in anti-de Sitter spacetime. We compute entanglement entropy of a nonlocal field theory in anti-de Sitter spacetime and find several interesting features. We find that area law is followed, but subleading terms are affected by nonlocality. We also find that the UV-finite term is universal. For the massless theory in three-dimensional anti-de Sitter spacetime we compute it exactly and find the novel feature that it shows oscillatory behavior.
\end{abstract}

DOI: $10.1103 /$ PhysRevD.99.125012

\section{INTRODUCTION}

Entanglement entropy is a very useful tool that helps us quantize the correlation between different localized subsystems of a quantum system. For local UV-finite theories entanglement entropy is known to follow an area law [1,2]. Explicit calculation in $d>2$ can be done for free field theories [3]. For field theories with holographic duals, entanglement entropy of a region $A$ can be computed holographically using the quantum corrected Ryu-Takayanagi formula [4,5].

$$
S_{\text {bdry }}(A)=\frac{\text { Area }_{\text {min }}}{4 G_{N}}+S_{\text {bulk }}+\cdots
$$

where Area $_{\min }$ denotes the minimal area surface in the bulk which ends in the boundaries of $A$. The ellipses refer to higher-order corrections in $1 / G_{N}$. The last term in the formula is the entanglement entropy in AdS. The entanglement entropy of fields in AdS is therefore an important component of the quantum corrected Ryu-Takayanagi formula and deserves further exploration. So far it has only been calculated for local free fields in AdS [6].

Nonlocal field theories present an interesting situation in this context. One would expect that the presence of nonlocal correlations in the theory to manifest itself in entanglement entropy. Indeed it is found that explicit nonlocality can have drastic effects on entanglement entropy including modification of area law in some cases

\footnotetext{
"gaunarain@gmail.com

nirmalyak@gmail.com
}

Published by the American Physical Society under the terms of the Creative Commons Attribution 4.0 International license. Further distribution of this work must maintain attribution to the author(s) and the published article's title, journal citation, and DOI. Funded by SCOAP.
[7-10], but not always [11-13]. It is therefore interesting to explore the behavior of entanglement entropy in field theories which have nonlocalities.

Nonlocal theories are quite interesting in their own and have been explored in a variety of contexts such as [14-18]. Nonlocal theories have also been explored in the context of black-hole information loss paradox [19,20].

In this paper, we compute entanglement entropy for a particular free nonlocal field theory. Our action is given by

$S_{N L}=\int \mathrm{d} x \sqrt{-g}\left[\frac{1}{2}(\partial \phi)^{2}+\frac{m^{2}}{2} \phi^{2}-\frac{\lambda^{2}}{2} \phi \frac{1}{-\square} \phi\right]$,

where we refer to $\lambda$ as the nonlocality scale. It has mass dimensions $[\mathrm{mass}]^{2}$. In flat spacetime where it is possible to do a momentum decomposition of fields, it should be noticed that such nonlocal terms will become important at small energy.

Apart from the general motivations given for computing entanglement entropy in nonlocal field theories, there is a further motivation for this theory. We have shown in a companion paper that these theories admit conformal field theory duals [21]. This calls for further investigation of these theories in a holographic context. Computation of entanglement entropy of this theory in AdS background would be a step towards a full understanding of the quantum corrected Ryu-Takayanagi formula (1) for these theories.

In this paper we consider odd dimensional anti-de Sitter spaces. The entanglement entropy for the theory (2) for generic odd dimensional AdS is computed using the replica trick and the heat kernel method. There are several interesting features. We find that the area law is followed but the subleading terms depend on the nonlocality scale. We find that there is a UV-finite universal term in entanglement entropy. For massless theories in $\mathrm{AdS}_{3}$ we 
explicitly compute it and show that it exhibits oscillatory behavior. This is a novel feature of nonlocal theories.

The paper is organized as follows. In Sec. II we study the nonlocal theory (2) in more detail. We calculate the Green's function for this theory in flat and AdS spacetime in Secs. II A and II B. In Sec. III we present our computation of entanglement entropy. We summarize our results in the conclusion in Sec. IV.

\section{NONLOCAL SCALAR FIELD}

In this section we consider a local scalar field theory consisting of two coupled fields. This leads to a nonlocal action once one of the fields gets decoupled from the system. Consider the following action for local field theory:

$S=\int \mathrm{d} x \sqrt{-g}\left[\frac{1}{2}(\partial \phi)^{2}+\frac{1}{2}(\partial \chi)^{2}+\frac{m^{2}}{2} \phi^{2}-\lambda \phi \chi\right]$,

where $\phi$ and $\chi$ are two scalar fields on curved nondynamical background, $\lambda$ is their coupling strength while $m$ is mass of scalar $\phi$. The equation of motion of two fields give $\left(-\square+m^{2}\right) \phi-\lambda \chi=0$ and $-\square \chi-\lambda \phi=0$. Integrating out $\chi$ from the second equation of motion yields $\chi=\lambda(-\square)^{-1} \phi$. This, when plugged back into the action (3), yields a massive nonlocal theory for scalar $\phi$ whose action is given by Eq. (2). This nonlocal action has issues of tachyon. In the simple case of flat spacetime it is noticed that the nonlocal piece in action reduces to $\phi(-p)\left(-\lambda^{2} / p^{2}\right) \phi(p)$ [where $\phi(p)$ is the Fourier transform of field $\phi(x)$ ]. This piece corresponds to something like tachyonic mass thereby resulting in issues of unitarity and instability of vacuum. Also, in the massless case, if we write $\phi_{1}=(\phi+\chi) / \sqrt{2}$ and $\phi_{1}=(\phi-\chi) / \sqrt{2}$, then the local action in Eq. (3) can be reduced to the action of two decoupled free scalar fields $\phi_{1}$ and $\phi_{2}$ of mass $\lambda$ and $-\lambda$ respectively. Immediately we notice the presence of tachyon field $\phi_{2}$. In the massive case things are just more involved, but the tachyonic issue remains. In what follows we will therefore consider $\lambda^{2} \rightarrow-\lambda^{2}$. This overcomes the issue of tachyons in the nonlocal theory. Also, it should be mentioned that the resulting nonlocal theory with $-\lambda^{2}$ can also arise as a low-energy limit of some ultraviolet complete theory in which sense it is an effective theory.

In this section we will study this simple free nonlocal theory on flat and AdS spacetime. In generic spacetime the Green's function equation is given by

$$
\left(-\square+m^{2}+\frac{\lambda^{2}}{-\square}\right) G\left(x, x^{\prime}\right)=-\frac{i \delta\left(x-x^{\prime}\right)}{\sqrt{-g}} .
$$

\section{A. Flat spacetime}

In this section we study this theory on flat spacetime. Understanding the behavior of theories in flat spacetime is important as in curved spacetime at ultralocal distances the theory behaves as in flat. This is also required for implementing suitable boundary conditions for the computation of the Green's function.

In flat spacetime one can use the momentum space representation to write the propagator. Writing $G\left(x, x^{\prime}\right)=$ $\int \mathrm{d} p /(2 \pi) \tilde{G}(p) \exp \left\{i p\left(x-x^{\prime}\right)\right\}$, it is seen that

$G_{f}^{\mathrm{NL}}\left(x, x^{\prime}\right)=-i \int \frac{\mathrm{d}^{d} p}{(2 \pi)^{d}}\left(p^{2}+m^{2}+\frac{\lambda^{2}}{p^{2}}\right)^{-1} e^{i p\left(x-x^{\prime}\right)}$

where the subscript in $G_{f}$ implies flat spacetime. The integrand can be integrated easily by making use of partial fraction and decomposing it. It can be written in an alternative manner as

$$
\begin{aligned}
\frac{1}{p^{2}+m^{2}+\lambda^{2} / p^{2}} & =\frac{p^{2}}{p^{4}+m^{2} p^{2}+\lambda^{2}} \\
& =\frac{A}{p^{2}+r_{-}^{2}}+\frac{B}{p^{2}+r_{+}^{2}},
\end{aligned}
$$

where

$$
r_{-}^{2}=\frac{m^{2}-\sqrt{m^{4}-4 \lambda^{2}}}{2} \quad r_{+}^{2}=\frac{m^{2}+\sqrt{m^{4}-4 \lambda^{2}}}{2},
$$

while $A=-r_{-}^{2} /\left(r_{+}^{2}-r_{-}^{2}\right)$ and $B=r_{+}^{2} /\left(r_{+}^{2}-r_{-}^{2}\right)$. For massless fields $\left(m^{2} \rightarrow 0\right) \quad r_{ \pm}^{2}= \pm i \lambda, \quad A=1 / 2, \quad$ and $B=1 / 2$. After the partial fraction each of the pieces can be further expressed by using the inverse Laplace transform. This allows us to write $\left(p^{2}+m^{2}+\lambda^{2} / p^{2}\right)^{-1}$ in the inverse Laplace form. Then one can express the flat spacetime Green's function in Eq. (5) as follows:

$$
\begin{aligned}
G_{f}^{\mathrm{NL}}\left(x, x^{\prime}\right)= & -i \int \frac{\mathrm{d}^{d} p}{(2 \pi)^{d}} \int_{0}^{\infty} \mathrm{d} s\left[\cosh (s \beta)-\frac{\alpha}{\beta} \sinh (s \beta)\right] \\
& \times e^{-s\left(p^{2}+\alpha\right)+i p\left(x-x^{\prime}\right)},
\end{aligned}
$$

where $\alpha=m^{2} / 2$ and $\beta=\sqrt{m^{4}-4 \lambda^{2}} / 2$. In the integrand one can smoothly take massless limits to obtain the Green's function for the nonlocal massless scalar field. In this massless limit $\alpha \rightarrow 0$ while $\beta \rightarrow i \lambda$, then the term in the square bracket in the integrand still remains real as $\cosh (s \beta) \rightarrow \cos (s \lambda)$ and $\sinh (s \beta) \rightarrow i \sin (s \lambda)$.

In Eq. (8) one can first evaluate the $p$ integration which is done by completing the square leading to a Gaussian type integral. In the process of completing the square for $p$, one encounters division by $s$. This implies that the lower limit of $s$ integration should be regularized by imposing a cutoff. One can then perform integration over $p$ easily leading to

$G_{f}^{\mathrm{NL}}\left(x, x^{\prime}\right)=\int_{\delta^{2}}^{\infty} \mathrm{d} s(4 \pi s)^{-d / 2} e^{-\mu^{2} / 4 s}\left(A e^{-s r_{-}^{2}}+B e^{-s r_{+}^{2}}\right)$, 
where $\mu\left(x, x^{\prime}\right)=\sqrt{\left(x-x^{\prime}\right)^{2}}$. This is a very subtle definite integral due to the lower limit of integration. This integral can be seen as a sum of two integrals of local theories with masses $r_{-}$and $r_{+}$, respectively. It is important to understand the singularity structure of this integral carefully as it will be seen to a play an important role later on. As a result we first consider the local field theory which can be obtained from above by taking the $\lambda \rightarrow 0$ limit. The above integral becomes the following:

$$
G_{f}^{\mathrm{L}}\left(x, x^{\prime}\right)=\int_{\delta^{2}}^{\infty} \mathrm{d} s(4 \pi s)^{-d / 2} e^{-\mu^{2} / 4 s} e^{-s m^{2}} .
$$

When $x \neq x^{\prime}(\mu \neq 0)$, the integral can be performed exactly in closed form for $\delta=0$. Even otherwise, it is seen that $G_{f}^{\mathrm{L}}$ has a Taylor series expansion around $\delta=0$. The leading term of which has a singularity which goes as $\sim \mu^{2-d}$. On the other hand, it is seen that performing first the small distance expansion and then doing the $s$ integration leads to no short-distance singularity in $G_{f}$. This indicates the subtlety associated with the point $(\delta, \mu)=(0,0)$. The standard rule we will follow is to first perform the $s$ integration and then extract the short-distance singularity.

Keeping this in mind Eq. (8) is evaluated by first performing the integration over $p$ then integration over $s$. For the $m=0$ case, the $s$ integral can be performed exactly in closed form in arbitrary dimensions. In four dimensions it acquires a simplified form and is given by

$$
G_{f}\left(x, x^{\prime}\right)=\frac{\sqrt{i \lambda} K_{1}(\sqrt{i \lambda} \mu)+\sqrt{-i \lambda} K_{1}(\sqrt{-i \lambda} \mu)}{8 \pi^{2} \mu} .
$$

In the case when $m \neq 0$ things are complicated. Here two possibilities arises $m^{4}>4 \lambda^{2}$ and $4 \lambda^{2}>m^{4}$, where both $m$ and $\lambda$ are positive. In the former case one can take limit $\lambda \rightarrow 0$ (locality limit), while in the later case one can take the limit $m \rightarrow 0$ (massless limit). In the locality limit, $r_{-}^{2}=0$ and $r_{+}^{2}=m^{2}$. In this case $A=0$ while $B=1$ $\left(\alpha=\beta=m^{2} / 2\right)$. In this case one gets the propagator for the local massive scalar field which is

$\left.G_{f}^{\mathrm{NL}}(\mu)\right|_{\lambda=0}=(2 \pi)^{-d / 2}\left(\frac{m}{\mu}\right)^{(d-2) / 2} K_{\frac{d}{2}-1}(m \mu)=G_{f}^{L}(\mu)$,

where $G_{f}^{L}(\mu)$ is the local Green's function of the free massive scalar field. For $m \neq 0$ one can still perform the integral in closed form. For the massive nonlocal scalar field in arbitrary spacetime dimension this Green's function $G_{f}$ is given by

$$
\begin{aligned}
G_{f}^{\mathrm{NL}}(\mu)= & \frac{(2 \pi)^{-d / 2} \mu^{1-d / 2}}{\left(r_{+}^{2}-r_{-}^{2}\right)} \\
& \times\left[r_{+}^{d / 2+1} K_{\frac{d}{2}-1}\left(r_{+} \mu\right)-r_{-}^{d / 2+1} K_{\frac{d}{2}-1}\left(r_{-} \mu\right)\right],
\end{aligned}
$$

where $r_{+}$and $r_{-}$are stated before. In fact one can express this Green's function as a sum of the Green's function for two local massive scalar fields with mass $r_{+}^{2}$ and $r_{-}^{2}$ and coefficient $A$ and $B$, respectively. This is expected and is clear from Eq. (9). As a result one can also write $G_{f}^{\mathrm{NL}}$ as

$$
G_{f}^{\mathrm{NL}}(\mu)=\left.A G_{f}^{L}(\mu)\right|_{r_{-}}+\left.B G_{f}^{L}(\mu)\right|_{r_{+}} .
$$

This will also be useful in fixing the appropriate boundary conditions for the Green's function on AdS. One can do a short-distance expansion of the flat spacetime Green's function to isolate the singular behavior of the propagator. In four dimensions the two singular contributions are

$$
G_{f}^{\text {sing }}=\frac{1}{4 \pi^{2} \mu^{2}}+\frac{m^{2}}{8 \pi^{2}} \log (\mu)+\cdots,
$$

where $\mu=\mu\left(x, x^{\prime}\right)$. These are singular behaviors in four dimensions at short distances. This will be true in other even dimensions where the leading singularity will be $\sim \mu^{2-d}$. In even and odd dimensions we have the shortdistance singularity structure as

$$
\begin{aligned}
\left.G_{f}^{\text {sing }}\right|_{\mathrm{d}=\mathrm{even}} & =a_{0} \mu^{2-d}+a_{1} \mu^{4-d}+\cdots a_{L} \log \mu, \\
\left.G_{f}^{\text {sing }}\right|_{\mathrm{d}=\mathrm{odd}} & =a_{0} \mu^{2-d}+a_{1} \mu^{4-d}+\cdots+\frac{a_{L}}{\mu} .
\end{aligned}
$$

The $\log \mu$ contribution in even dimensions is also singular though in the infrared limit $(\mu \rightarrow \infty)$ it has a well-defined behavior. In massive theories one also has an inbuilt infrared cutoff $\Lambda_{\mathrm{IR}} \sim 1 / m$, which truncates the IR modes of the theory. Then it is noticed that the $\log \mu$ approaches $\log \Lambda_{\mathrm{IR}}=$ $-\log m$ in IR. This implies that the $\log$ contribution in $G_{f} \sim-m^{2} \log m$ which has a smooth massless limit.

\section{B. Anti-de Sitter}

In this section we will investigate the theory equation (2) in Euclidean AdS (EAdS) background. The $n$-dimensional EAdS spacetime $\mathbb{H}^{n}$ can be identified with the real uppersheeted hyperboloid in $(d+1)$ Minkowski spacetime $M_{d+1}$ : $X_{d}=\left\{x \in \mathbb{R}^{d+1}, x_{1}^{2}+\cdots+x_{d}^{2}-x_{d+1}^{2}=-H^{-2}\right\}$, where $H$ is related to EAdS length. If the two points are denoted by $x$ and $x^{\prime}$, the length of geodesic connecting them is $\mu\left(x, x^{\prime}\right)$. It is useful to introduce a quantity $z\left(x, x^{\prime}\right)=\cosh ^{2}(H \mu / 2)$. For timelike distances $\mu^{2}<0$ which corresponds to $0 \leq$ $z<1$, while spacelike separation $0<\mu^{2}<\infty$ corresponds to $1<z<\infty . z=1$ is the lightlike separation. On AdS background the Green's function will be entirely a function of $\mu$ [or $\left.z\left(x, x^{\prime}\right)\right]$. This allows one to compute the Green's function exactly by solving linear differential equations.

In the case of nonlocal theory, the Green's function equation is given in Eq. (4). Before we solve for $G\left(x, x^{\prime}\right)$ on AdS, we first note the following important identities on curved spacetime. 
$-\left[\square^{2}-m^{2} \square+\lambda^{2}\right]^{-1} \square=A\left(-\square+r_{-}^{2}\right)^{-1}+B\left(-\square+r_{+}^{2}\right)^{-1}$,

where $A, B, r_{-}^{2}$, and $r_{+}^{2}$ have the same values as in flat spacetime. If we multiply both sides by the operator

$$
\left[\square^{2}-m^{2} \square+\lambda^{2}\right]=\left(-\square+r_{-}^{2}\right)\left(-\square+r_{+}^{2}\right),
$$

then we clearly note that it is identically satisfied. It is this identification which allows us to compute the Green's function of the nonlocal theory in anti-de Sitter spacetime. This means that the full Green's function for the nonlocal theory $G_{\mathrm{NL}}\left(x, x^{\prime}\right)$ is a sum of two Green's functions

$$
G_{\mathrm{NL}}\left(x, x^{\prime}\right)=A G_{-}\left(x, x^{\prime}\right)+B G_{+}\left(x, x^{\prime}\right),
$$

where

$$
\left(-\square+r_{-}^{2}\right) G_{-}\left(x, x^{\prime}\right)=0, \quad\left(-\square+r_{+}^{2}\right) G_{+}\left(x, x^{\prime}\right)=0
$$

are the two Green's function on AdS which are determined by requiring that the short-distance singularity of AdS propagator matches with the singular behavior of the flat spacetime propagator. This just gives the usual AdS propagator of the free local massive scalar field which is well known in the literature [22-25]. For example, for local massive scalar field on AdS we have $\left(-\square+m^{2}\right) G\left(x, x^{\prime}\right)=0$ as the Green's function equation. Then $G\left(x, x^{\prime}\right)$ follows

$$
z(1-z) G^{\prime \prime}+[c-(a+b+1) z] G^{\prime}-a b G=0,
$$

where $a=\left[d-1+\left((d-1)^{2}+4 m^{2} / H^{2}\right)^{1 / 2}\right] / 2, b=[d-$ $\left.1-\left((d-1)^{2}+4 m^{2} / H^{2}\right)^{1 / 2}\right] / 2$ and $c=d / 2$. This hypergeometric differential equation has two linearly independent solutions: $z^{-a}{ }_{2} F_{1}\left(a, a-c+1, a-b+1 ; z^{-1}\right)$ and $z^{-b}{ }_{2} F_{1}\left(b, b-c+1, b-a+1 ; z^{-1}\right)$ [22-26]. As $a>0$ and $b<0$, therefore, the former solution falls off at the boundary of AdS while the latter does not. This allows one to pick the former. By requiring the short-distance singularity of the AdS propagator to match with the singular behavior of the flat spacetime propagator one concludes that $G(z)=q \times z^{-a}{ }_{2} F_{1}\left(a, a-c+1, a-b+1 ; z^{-1}\right)$. The coefficient $q$ is fixed by requiring that the strength of the singularity of the AdS propagator matches with the strength of the singularity in flat spacetime. This gives

$$
q=\frac{(4 \pi)^{(1-d) / 2} 2^{-(a-b+1)} H^{d-2} \Gamma(a)}{\Gamma(a-c+3 / 2)} .
$$

Using this one can write the Green's function $G_{N L}$ for the nonlocal scalar on AdS to be

$$
\begin{aligned}
G_{\mathrm{NL}}(z)= & A q_{-} z^{-a_{-}}{ }_{2} F_{1}\left(a_{-}, a_{-}-c_{-}+1, a_{-}-b_{-}+1 ; z^{-1}\right) \\
& +B q_{+} z^{-a_{+}}{ }_{2} F_{1}\left(a_{+}, a_{+}-c_{+}+1, a_{+}-b_{+}+1 ; z^{-1}\right),
\end{aligned}
$$

where the coefficients $q_{-}$and $q_{+}$are determined from Eq. (22) with mass replaced by $r_{-}$and $r_{+}$, respectively, while $A$ and $B$ are the same as previously mentioned. The parameters of the hypergeometric functions are given by

$$
\begin{array}{ll}
a_{-}=\frac{1}{2}\left(d-1+\sqrt{(d-1)^{2}+\frac{4 r_{-}^{2}}{H^{2}}}\right), & b_{-}=\frac{1}{2}\left(d-1-\sqrt{(d-1)^{2}+\frac{4 r_{-}^{2}}{H^{2}}}\right), \\
a_{+}=\frac{1}{2}\left(d-1+\sqrt{(d-1)^{2}+\frac{4 r_{+}^{2}}{H^{2}}}\right), & b_{+}=\frac{1}{2}\left(d-1-\sqrt{(d-1)^{2}+\frac{4 r_{+}^{2}}{H^{2}}}\right),
\end{array}
$$

and $c_{-}=c_{+}=d / 2$. The parameters $q_{-}$and $q_{+}$are given by

$$
q_{-}=\frac{2^{-\left(a_{-}-b_{-}+1\right)} H^{d-2} \Gamma\left(a_{-}\right)}{(4 \pi)^{(d-1) / 2} \Gamma\left(a_{-}-c_{-}+3 / 2\right)}, \quad q_{+}=\frac{2^{-\left(a_{+}-b_{+}+1\right)} H^{d-2} \Gamma\left(a_{+}\right)}{(4 \pi)^{(d-1) / 2} \Gamma\left(a_{+}-c_{+}+3 / 2\right)} .
$$

The Green's function is analytic in $\lambda$. By taking $\lambda \rightarrow i \lambda$ one obtains the Green's function for the theory with tachyons. The Green's function remains well defined even when this transformation is done. The massless limit can be smoothly taken on AdS irrespective of nonlocality. In the massless limit $r_{+}^{2} \rightarrow i \lambda$ while $r_{-}^{2} \rightarrow-i \lambda$, while $A \rightarrow 1 / 2$ and $B \rightarrow$ $1 / 2$. Correspondingly, the parameters of hypergeometric functions can be obtained (see [27] for the nonlocal Green's function in de Sitter).

In Fig. 1 we plot this Green's function for various values of $\lambda_{s}$ for massless theory. It is seen that nonlocality starts to have an effect on the propagator at short distances. For the case of nonzero mass, the propagator has one more parameter; however, the structure remains the same. 


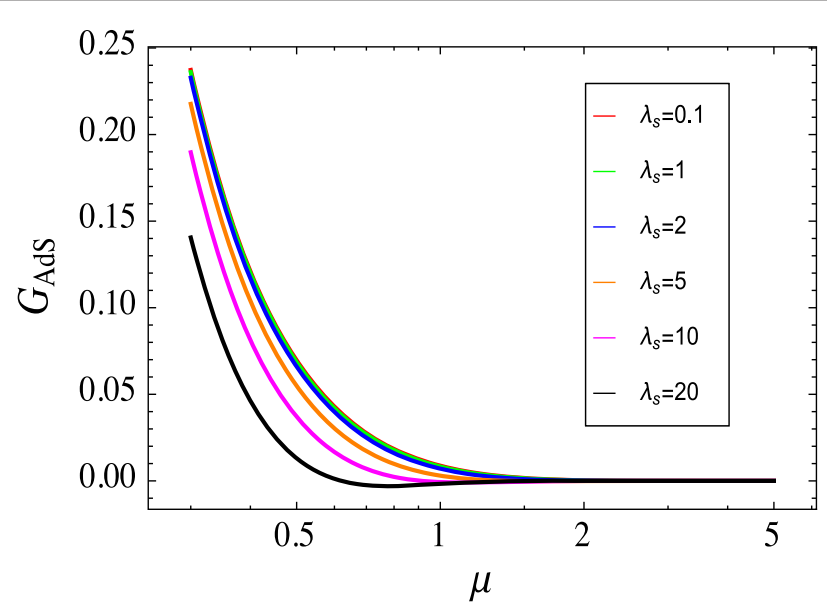

FIG. 1. The Green's function of massless nonlocal scalar field in four spacetime dimensions. The propagator is plotted for various strength of nonlocality $\lambda$ against the spacelike separation $\mu\left(x, x^{\prime}\right)$.

Here we have three cases (as in flat spacetime): $m^{2}<2 \lambda$, $m^{2}=2 \lambda$, and $m^{2}>2 \lambda$. In each case the propagator is real for spacelike separation. It is worthwhile to plot the propagator for a fixed value of $\lambda_{s}$ and for decreasing mass. It is seen that as $m \rightarrow 0$, the massive propagator smoothly approaches the massless nonlocal propagator. In Fig. 2 we plot this scenario. The case of equality is interesting as then the propagator depends only on one parameter. Physically it implies that the two length scales are comparable. In this case the nonlocality scale is coupled with the mass, so $m \rightarrow 0$ implies $\lambda \rightarrow 0$ simultaneously. The infrared limit of mass going to zero is well defined and smooth. In this limit the nonlocal Green's function is independent of any parameters in the theory (depending only on $H$ ). It is given by

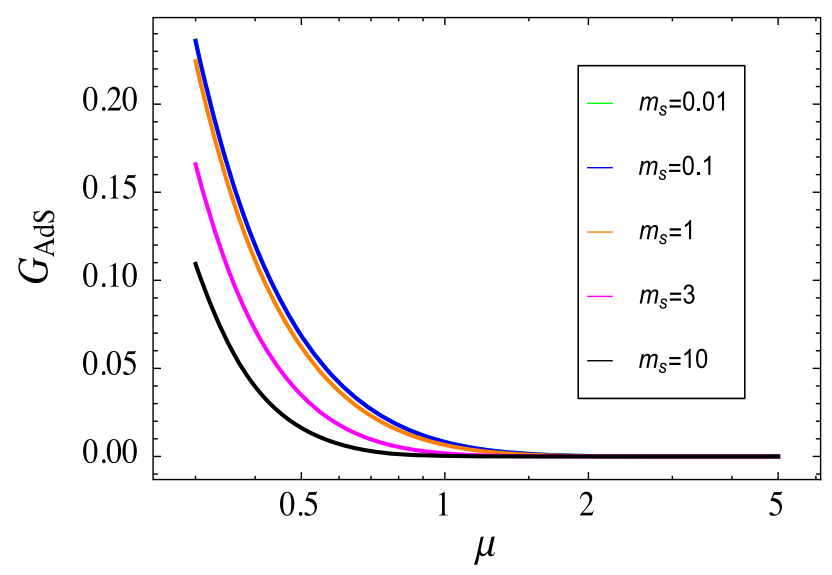

FIG. 2. The Green's function of the massive nonlocal scalar field in four spacetime dimensions. The propagator is plotted for various masses $m_{s}$ and for fixed $\lambda_{s}=1$, against the spacelike separation $\mu\left(x, x^{\prime}\right)$.

$$
\left.G_{\mathrm{NL}}\right|_{m^{2}=2 \lambda}=\frac{H^{2}\left[2 z+2(z-1) z \log \left(\frac{z-1}{z}\right)-1\right]}{16 \pi^{2}(z-1) z} .
$$

On doing the short-distance expansion $\mu \rightarrow 0$ it is noticed that the leading singular behavior of $G_{\mathrm{AdS}}(\mu)$ is exactly the same as in flat spacetime, which is expected due to boundary conditions. Besides this there is a next-toleading singular piece in four spacetime dimensions. This term is log divergent and is given by $\left(\left(2 H^{2}+m^{2}\right) /\right.$ $\left.8 \pi^{2}\right) \log \mu$. Compared to flat spacetime, here there is an extra contribution from $H$. Again, this term does not depend on the nonlocality strength $\lambda$. This again is not problematic in IR as argued in flat spacetime. Beside this there will also be terms like $\mu \log \mu$ which will appear in the higher-order expansion of the Green's function. Terms like these are not UV singular in the short-distance limit. It is noted that in the short-distance expansion one can arrange the various terms as the following series:

$$
\begin{aligned}
G_{\mathrm{NL}}= & -\frac{\pi^{1-d / 2} 2^{-d} \csc (d \pi / 2)}{H^{d-2} \Gamma(2-d / 2)} \frac{1}{(z-1)^{d / 2-1}}+\frac{E_{2}}{(z-1)^{d / 2-2}} \\
& +\cdots+E_{0},
\end{aligned}
$$

where $E_{0}$ is a constant piece in $z$ depending on mass $m$, nonlocality strength $\lambda, H$ and dimension $d$. This is a kind of universal piece as it is present in the expression of $G_{\mathrm{NL}}$ for all $z$. This term is given by

$E_{0}=\frac{H^{d-2} \pi^{1-d / 2} \csc (d \pi / 2)}{2^{d} \Gamma(d / 2)\left(r_{+}^{2}-r_{-}^{2}\right)}\left[\frac{r_{+}^{2} \Gamma\left(a_{+}\right)}{\Gamma\left(1-b_{+}\right)}-\frac{r_{-}^{2} \Gamma\left(a_{-}\right)}{\Gamma\left(1-b_{-}\right)}\right]$.

\section{ENTANGLEMENT ENTROPY}

In this section we compute the entanglement entropy (EE) in the AdS background using the replica trick [28-31]. The replica trick is the covariant formulation of the computation of either EE or Renyi entropy via usage of the effective action of the theory which is computed on a background with a conical defect.

The actual starting point is density matrix $\rho$. Any space can be divided by a surface $\Sigma$ into two parts: $A$ and $\bar{A}$. The field modes resides on both sides of the boundary surface $\Sigma$ (the projection of this on boundary is $B$ ). This has been depicted in Fig. 3. This leads to a Hilbert space factorization: $H=H_{A} \otimes H_{\bar{A}}$. Then tracing out the degree of freedom on $\bar{A}$ results in a reduced density matrix: $\rho_{A}=\operatorname{Tr}[|\psi\rangle\langle\psi|]$, where $\bar{\psi}$ is a ground state. The entanglement entropy $S_{A}$ will be defined as $S_{A}=-\operatorname{Tr}\left(\rho_{A} \log \rho_{A}\right)$. In order to compute EE for field theoretic systems one has to make use of the replica trick, which makes use of pathintegral formalism. In this one, we start by expressing the density matrix in the path-integral form. Following [4] we first define a vacuum state of QFT by a path integral over 


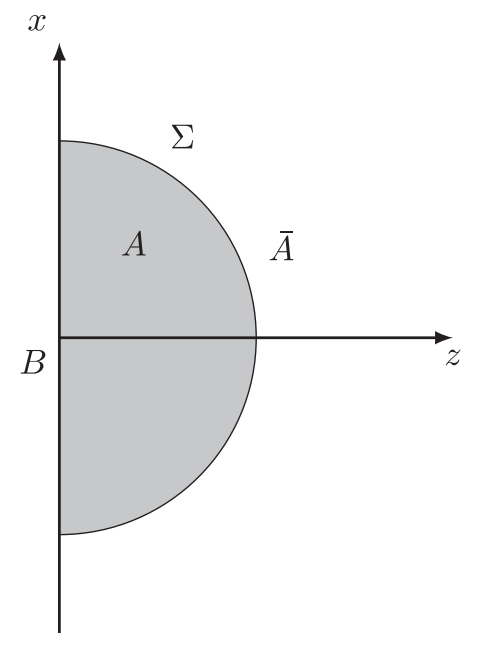

FIG. 3. Minimal surface corresponding to region $B$ in the boundary. The $z$ direction denotes the bulk while the $x$ represents the spatial coordinate on boundary. The region surrounded by $B$ is $\Sigma$ and is denoted by $A$.

half of the total Euclidean space $\tau \leq 0$, in such a manner that the quantum field takes a fixed boundary condition $\psi(\tau=0, x)=\psi_{0}(x)$. Then we have

$$
\Psi\left[\psi_{0}(x)\right]=\int_{\psi(\tau=0, x)=\psi_{0}(x)} \mathcal{D} \psi \exp (-I),
$$

where $I$ is the Euclidean action of the field $\psi$ and $\Psi$ is a functional of boundary $\psi_{0}$. The direction of $x$ is orthogonal to boundary $\Sigma$. The surface defined by $x=0, \tau=0$ naturally divides the surface $\Sigma$ in two parts: $x>0$ and $x<0$. Correspondingly, the boundary data gets separated as $\psi_{ \pm}(x)=\psi_{0}(x)$ for $x>0$ and $x<0$, respectively. Tracing out over $\psi_{-}$results in a reduced density matrix

$$
\rho\left(\psi_{+}^{1}, \psi_{+}^{2}\right)=\int \mathcal{D} \psi_{-} \Psi\left(\psi_{+}^{1}, \psi_{-}\right) \Psi\left(\psi_{+}^{2}, \psi_{-}\right),
$$

where the path integral goes over the whole Euclidean spacetime except the cut at $\tau=0, x>0$, while the field $\psi(A)$ takes the value $\psi_{+}^{2}$ above the cut and $\psi_{+}^{1}$ below the cut. The quantity $\operatorname{Tr} \rho^{n}$ is then defined over the $n$-sheeted copy of the cut spacetime, where appropriate analytic gluing is done when passing from one sheet to another. This quantity is given by

$$
\operatorname{Tr} \rho^{n}=\frac{Z_{n}}{Z_{1}^{n}}
$$

where $Z_{n}$ is the path integral over the $n$-sheeted covering space $\mathcal{M}^{n}$ which is obtained by analytically sewing $n$ copies of the original Euclidean manifold $\mathcal{M}$ along the cut. The EE and Renyi entropy are correspondingly given by
$S_{E E}=\lim _{n \rightarrow 1}\left(n \partial_{n}-1\right) W_{n}, \quad S_{n}=\lim _{n \rightarrow 1} \frac{W_{n}-n W_{1}}{n-1}$,

where $W_{n}=-\log Z_{n}$ is the effective action on $\mathcal{M}^{n}$ while $W_{1}=\log Z_{1}$ is the usual effective action on $\mathcal{M}$.

The effective action $W_{1}$ (where 1 indicates the EA has been computed on the single copy of manifold $\mathcal{M}$ ) for the field theory can be computed using the standard field theory methods in flat spacetime. On curved space there are limitations as the usual Feynman perturbation theory cannot be directly extended. However, one can still perform some computations on maximally symmetric background or one-loop approximation. In the later case, the effective action $W_{1}$ to one-loop can be expressed using the heat kernel of the Hessian of the theory on the curved background.

$$
\begin{aligned}
W & =-\frac{1}{2} \int_{0}^{\infty} \frac{\mathrm{d} s}{s} \operatorname{Tr} K(x, x ; s) \\
& =-\lim _{x \rightarrow x^{\prime}} \frac{1}{2} \int_{0}^{\infty} \frac{\mathrm{d} s}{s} \operatorname{Tr}\left\langle x|\exp (-s \triangle)| x^{\prime}\right\rangle,
\end{aligned}
$$

where $K\left(x, x^{\prime} ; s\right)$ is the heat kernel of the operator $\triangle$, while $x$ and $x^{\prime}$ are two spacetime points and Tr is over all Lorentz and spacetime indexes. The lower limit of the $s$ integral is not well defined and leads to UV divergences which also arise due to the co-incident limit. In fact the integrand is singular at $s=0$. One has to impose a cutoff which corresponds to imposing an ultraviolet cutoff in theory. This singles out precisely the divergent part of the above integral leaving behind a UV-finite piece.

In the replica method, the entropy is computed by first obtaining the effective action on the background of $n$ manifold $\left(\mathcal{M}^{n}\right)$. This can be computed by exploiting the known results of the heat kernel of the operator on the background of the cone [13,32-34] and making use of the Sommerfeld formula [35] which expresses the heatkernel modification on the manifold with the conical singularity as

$$
\begin{aligned}
K_{n}\left(\phi, \phi^{\prime} ; s\right)= & K_{1}\left(\phi, \phi^{\prime} ; s\right)+\frac{i}{4 \pi n} \int_{\Gamma} \mathrm{d} \omega \cot \left(\frac{\omega}{2 n}\right) \\
& \times K_{1}\left(\phi-\phi^{\prime}+\omega ; s\right),
\end{aligned}
$$

where $\phi$ is the angle and other coordinates have been skipped; the contour $\Gamma$ consists of two lines: one goes from $(-\pi+i \infty)$ to $(-\pi-i \infty)$ intersecting the real axis between poles $-2 \pi n$ and 0 of $\cot (\omega / 2 n)$, and another goes from $(\pi-i \infty)$ to $(\pi+i \infty)$ intersecting the real axis between poles 0 and $2 \pi n$. For $n=1$, the integrand is $2 \pi$ periodic and the contribution from the two vertical lines cancel each other.

This modification of the heat kernel on $\mathcal{M}^{n}$ gets reflected on the computation of the one-loop effective action and the Green's function on $\mathcal{M}^{n}$, and allows us 
to do the computation of the entropy via the replica trick. In the case of AdS, one then has to go to a coordinate system where it is possible to successfully implement the procedure of having $n$ copies of the manifold where gluing is done analytically to construct $\mathcal{M}^{n}$. The maximally symmetric EAdS can be represented as an embedding of surface

$$
x_{1}^{2}+\cdots+x_{d}^{2}-x_{d+1}^{2}=-H^{-2}
$$

in $\mathbb{R}^{1, d}$ with metric

$$
\mathrm{d} s^{2}=\mathrm{d} x_{1}^{2}+\cdots+\mathrm{d} x_{d}^{2}-\mathrm{d} x_{d+1}^{2} .
$$

Under the transformation $x_{d+1}+x_{d}=z^{-1} H^{-2}, \quad x_{a}=$ $(H z)^{-1} y_{a}$, the induced metric on the above hyperboloid then becomes a standard $\operatorname{AdS}_{d}$ metric:

$$
\mathrm{d} s^{2}=(H z)^{-2}\left(\mathrm{~d} z^{2}+\eta_{a b} \mathrm{~d} y^{a} \mathrm{~d} y^{b}\right) .
$$

If one polar transforms $z$ and $y$, implying $z=r \sin \theta$ and $y=r \cos \theta$, then the entangling surface $\Sigma$ is given by $r=$ $r_{0}$ and $t_{E}=0 . E \mathrm{AdS}_{d}$ has another useful foliation given by

$$
\begin{aligned}
x_{d+1} & =\sqrt{\rho^{2}+L^{2}} \cosh u, \quad x_{1}=\rho \sin (\tau / L), \quad x_{d}=\rho \cosh (\tau / L), \\
x_{2} & =\sqrt{\rho^{2}+L^{2}} \sinh (u) \cos \left(\phi_{1}\right), \quad x_{3}=\sqrt{\rho^{2}+L^{2}} \sinh (u) \sin \left(\phi_{1}\right) \cos \left(\phi_{2}\right), \ldots, \\
x_{d-2} & =\sqrt{\rho^{2}+L^{2}} \sinh (u) \sin \left(\phi_{1}\right) \sin \left(\phi_{2}\right) \cdots \sin \left(\phi_{d-2}\right),
\end{aligned}
$$

where $L=H^{-1}[36,37]$. This transformation expresses the EAdS spacetime as a Euclidean topological black hole whose horizon is hyperbolic space $H^{d-2}$. This metric is given by

$$
\begin{aligned}
\mathrm{d} s^{2}= & \frac{\mathrm{d} \rho^{2}}{1+H^{2} \rho^{2}}+H^{2} \rho^{2} \mathrm{~d} \tau_{E}^{2} \\
& +\left(H^{-2}+\rho^{2}\right)\left(\mathrm{d} v^{2}+\sinh ^{2} v \mathrm{~d} \Omega_{d-3}^{2}\right),
\end{aligned}
$$

where the horizon occurs at $\rho=0$ having a uniform negative curvature. This topological black hole has a horizon temperature $T=H /(2 \pi)$. The entangling surface $\Sigma$ corresponds to this horizon at $\tau_{E}=0$. We represent the area of this entangling surface by $\mathcal{A}(\Sigma)$ which has been computed in $[6,36]$. For the $n$ copies of this manifold, the covering space $\mathcal{M}^{n}$ has the period $\tau_{E} \sim \tau_{E}+2 n \pi / H$. The partition function $Z_{n}$ acquires a thermal nature with temperature $H /(2 n \pi)$.

On the AdS geometry, which is maximally symmetric, one can compute the heat kernel $K_{1}\left(x, x^{\prime} ; s\right)$ exactly [38], using the one which can compute the effective action by making use of the relation equation (33). This can then be used to compute the entropy. The effective action involves the trace of the heat kernel. Using the above coordinate system equation (39) the trace of the heat kernel is given by

$$
\begin{aligned}
\operatorname{Tr} K_{n}(s) & =H^{3-d} \int_{0}^{2 \pi n / H} \mathrm{~d} \tau_{E} \int_{H^{d-2}} \mathrm{~d} V_{d-2} \int_{0}^{\infty} \mathrm{d} \rho \rho\left(1+H^{2} \rho^{2}\right)^{\frac{d-3}{2}} K_{n}(x, x ; s) \\
& =n \operatorname{Tr} K_{1}(s)+\frac{i}{2} \frac{\mathcal{A}(\Sigma)}{H^{d-2}} \int_{0}^{\infty} \mathrm{d} \rho \rho\left(1+H^{2} \rho^{2}\right)^{\frac{d-3}{2}} \int_{\Gamma} \mathrm{d} \omega \cot \left(\frac{\omega}{2 n}\right) K_{1}(\mu ; s),
\end{aligned}
$$

where

$$
z\left(x, x^{\prime}\right)=\cosh ^{2}\left(\frac{H \mu}{2}\right)=1+(H \rho)^{2} \sin ^{2}\left(H\left(\tau_{E}-\tau_{E}^{\prime}\right)\right)=1+(H \rho)^{2} \sin ^{2}(\omega / 2)
$$

is the EAdS invariant quantity for the two points $x$ and $x^{\prime}$ which differ only in the $\tau_{E}$ direction for the EAdS metric stated in Eq. (39), while $\mu\left(x, x^{\prime}\right)$ is the geodesic distance between $x$ and $x^{\prime}$. The expression for the trace of the heat kernel immediately gives us the expression for the effective action on $\mathcal{M}^{n}$ using Eq. (33) which can be used to compute entropy. The expression of the effective action is given by

$$
W_{n}=n W_{1}-\frac{i}{2} \frac{\mathcal{A}(\Sigma)}{H^{d-2}} \int_{\delta^{2}}^{\infty} \frac{\mathrm{d} s}{s} \int_{0}^{\infty} \mathrm{d} \rho \rho\left(1+H^{2} \rho^{2}\right)^{\frac{d-3}{2}} \int_{\Gamma} \mathrm{d} \omega \cot \left(\frac{\omega}{2 n}\right) K_{1}(\mu ; s)
$$

The EE (or Renyi) can be computed following Eq. (32) which is then given by 


$$
\begin{gathered}
S_{\mathrm{EE}}=\lim _{n \rightarrow 1}-\frac{-i \mathcal{A}(\Sigma)}{2 H^{d-2}}\left(n \partial_{n}-1\right) F(n), \\
S_{\text {Renyi }}=\lim _{n \rightarrow 1}-\frac{i}{2(n-1)} \frac{\mathcal{A}(\Sigma)}{H^{d-2}} F(n),
\end{gathered}
$$

where

$$
\begin{aligned}
F(n)= & \int_{\delta^{2}}^{\infty} \frac{\mathrm{d} s}{s} \int_{0}^{\infty} \mathrm{d} \rho \rho\left(1+H^{2} \rho^{2}\right)^{\frac{d-3}{2}} \int_{\Gamma} \mathrm{d} \omega \cot \left(\frac{\omega}{2 n}\right) \\
& \times K_{1}(\mu ; s)
\end{aligned}
$$

$\cosh ^{2}\left(\frac{H \mu}{2}\right)=1+(H \rho)^{2} \sin ^{2}(\omega / 2)$ and $K_{1}(\mu ; s)$ is the EAdS heat kernel on manifold $\mathcal{M}$ [38]. This is the exact expression for the EE and Renyi entropy, and it goes as the area of the surface $\Sigma$ which is denoted above by $\mathcal{A}(\Sigma)$.

The integral stated in Eq. (45), however, is not easy to compute. It consists of three integrals: one over $\rho$ which can be traded to integral over $\mu$ using the relation $\cosh ^{2}\left(\frac{H \mu}{2}\right)=$ $1+(H \rho)^{2} \sin ^{2}(\omega / 2)$, the second is a contour integral over $\omega$ for contour $\Gamma$, while the last integral is over $s$. In the former one can do the transformation of variables from $\rho$ to $\mu$. This leads to the following expression for $F(n)$ :

$$
\begin{aligned}
F(n)= & \frac{1}{4 H} \int_{\delta^{2}}^{\infty} \frac{\mathrm{d} s}{s} \int_{\Gamma} \mathrm{d} \omega \frac{\cot (\omega / 2 n)}{\sin ^{2}(\omega / 2)} \int_{0}^{\infty} \mathrm{d} \mu \sinh (H \mu) \\
& \times\left[1+\frac{\sinh ^{2}(H \mu / 2)}{\sin ^{2}(\omega / 2)}\right]^{(d-3) / 2} K_{1}^{d}(\mu ; s)
\end{aligned}
$$

where $K_{1}^{d}(\mu ; s)$ is the heat kernel on the AdS metric which is well known from the literature [38]. This integral is not easy to perform in arbitrary dimensions; however, in odd dimensions the expression in square brackets becomes a polynomial in $\sinh ^{2}(H \mu / 2)$ when expanded. This offers some simplicity in obtaining expressions for entropy in odd dimensions.

\section{A. Nonlocal heat kernel and effective action}

The quantity that enters the computation of the entropy is the effective action. In this sun section we will compute the effective action of the theory considered here by making use of the heat kernel on the AdS. For the free nonlocal theory considered in Eq. (2) (with the negative $-\lambda^{2}$ ) the effective action is given by

$$
\begin{aligned}
W= & -\log Z=\frac{1}{2} \log \operatorname{det}\left(-\square+m^{2}+\frac{\lambda^{2}}{-\square}\right) \\
= & \frac{1}{2} \log \operatorname{det}\left[(-\square)^{-1}\left(\square^{2}-m^{2} \square+\lambda^{2}\right)\right], \\
= & -\frac{1}{2} \log \operatorname{det}(-\square)+\frac{1}{2} \log \operatorname{det}\left(-\square+r_{-}^{2}\right) \\
& +\frac{1}{2} \log \operatorname{det}\left(-\square+r_{+}^{2}\right),
\end{aligned}
$$

where, in obtaining last line, we have used Eq. (18) and $r_{-}^{2}$ and $r_{+}^{2}$ are given in Eq. (7), respectively. Each of the piece in the last line of Eq. (47) can be expressed in terms of the heat kernel of the $\square$ operator on AdS which gives the following expression for the effective action of our theory:

$W_{1}=\lim _{x \rightarrow x^{\prime}} \frac{1}{2} \int_{\delta^{2}}^{\infty}\left(-\frac{\mathrm{d} s}{s}\right)\left(e^{-s r_{+}^{2}}+e^{-s r_{-}^{2}}-1\right) \operatorname{Tr} K_{0}\left(x, x^{\prime} ; s\right)$,

where $K_{0}\left(x, x^{\prime} ; s\right)=\left\langle x\left|e^{-s(-\square)}\right| x^{\prime}\right\rangle$ is the heat kernel of the $\square$. Equation (48) tell us that $K_{1}$ appearing in Eq. (46) is given by

$$
K_{1}\left(x, x^{\prime} ; s\right)=\left(e^{-s r_{+}^{2}}+e^{-s r_{-}^{2}}-1\right) K_{0}\left(x, x^{\prime} ; s\right) .
$$

In the case when nonlocality strength goes to zero $(\lambda \rightarrow 0)$ one recovers the heat kernel of the local massive scalar field operator as $r_{-}^{2} \rightarrow 0$. The heat kernel $K_{0}$ on the AdS background is known in closed form exactly [38] and is different in odd and even dimensions. For the AdS geometry it is given by

$$
\begin{aligned}
K_{0}^{\text {odd }}= & \left(\frac{-1}{2 \pi}\right)^{\frac{d-1}{2}}(4 \pi s)^{-1 / 2}\left[\frac{H}{\sinh (H \mu)} \frac{\partial}{\partial \mu}\right]^{\frac{d-1}{2}} \\
& \times \exp \left[-\frac{\mu^{2}}{4 s}-\frac{(d-1)^{2} H^{2} s}{4}\right], \\
K_{0}^{\text {even }}= & \left(\frac{-1}{2 \pi}\right)^{\frac{d-2}{2}} \frac{\sqrt{2} e^{-(d-1)^{2} H^{2} s / 4}}{(4 \pi s)^{3 / 2}}\left[\frac{H}{\sinh (H \mu)} \frac{\partial}{\partial \mu}\right]^{\frac{d-2}{2}} \\
\times & \int_{H \mu}^{\infty} \mathrm{d} \mu^{\prime} \frac{H^{2} \mu^{\prime} e^{-\mu^{\prime 2} / 4 s}}{\sqrt{\cosh \left(H \mu^{\prime}\right)-\cosh (H \mu)}} .
\end{aligned}
$$

These can then be plugged into Eq. (49) to obtain the expression for $K_{1}$.

\section{B. $\mathbf{A d S}_{3}$}

We first consider the case of three-dimensional AdS space. By making use of the heat kernel for $K_{0}$ given in Eq. (50) and using the expression for $K_{1}$ from Eq. (49) one can obtain $K_{1}$ for three-dimensional AdS to be 


$$
K_{1}^{3}(\mu ; s)=\frac{H \mu\left[2 e^{-m^{2} s / 2} \cosh \left(s \sqrt{m^{4}-4 \lambda^{2}} / 2\right)-1\right] \exp \left(-H^{2} s-\mu^{2} / 4 s\right)}{(4 \pi s)^{3 / 2} \sinh (H \mu)}
$$

This is the kernel for the nonlocal theory considered here. The kernel for the local massive theory can be obtained by taking the limit $\lambda \rightarrow 0$ which matches with the expression in [38-40]. Using the Sommerfeld formula stated in Eq. (34) one can compute the kernel on $\mathcal{M}^{n}$. In the computation of entropy we are, however, interested in quantity $F(n)$ which is mentioned in Eq. (46) and uses the heat kernel. In 3D both the entanglement and Renyi (in the limit $n \rightarrow 1$ ) entropy are same. It is given by

$$
\begin{aligned}
S_{\mathrm{EE}}= & S_{\text {Renyi }}=\frac{2 \mathcal{A}^{1}(\Sigma)}{3 \sqrt{\pi}} \\
& \times\left[\frac{1}{\delta}+\sqrt{\pi}\left(H-\sqrt{H^{2}+r_{-}^{2}}-\sqrt{H^{2}+r_{+}^{2}}\right)+\cdots\right],
\end{aligned}
$$

where $\mathcal{A}^{1}(\Sigma)$ is the area of the surface in three dimensions. The leading term is the UV divergent piece which diverges in the limit $\delta \rightarrow 0$ while the second term is the finite term. Terms of order $\mathcal{O}(\delta)$ have been ignored as they do not survive in the UV limit. The leading divergent piece is independent of the mass or nonlocality present in the theory; however, the finite piece contains information about both mass and nonlocality. This finite piece is analytic in $m$ and $\lambda$, and is always real. In particular, one can write the finite piece in $3 \mathrm{D}$ as follows,

$$
\begin{aligned}
S_{E E}^{\text {finite }}= & \frac{2 \mathcal{A}^{1}(\Sigma)}{3}\left(H-\sqrt{H^{2}+r_{-}^{2}}-\sqrt{H^{2}+r_{+}^{2}}\right) \\
= & \frac{2 \mathcal{A}^{1}(\Sigma)}{3}\left(H-\sqrt{u} e^{-v / 2}-\sqrt{u} e^{v / 2}\right)=\frac{2 \mathcal{A}^{1}(\Sigma)}{3} \\
& \times(H-2 \sqrt{u} \cosh (v / 2)),
\end{aligned}
$$

where $u=\sqrt{H^{4}+m^{2} H^{2}+\lambda^{2}}$ and $\tanh v=\sqrt{m^{4}-4 \lambda^{2}}$ / $\left(2 H^{2}+m^{2}\right)$. If $v$ changes sign or becomes imaginary, still the entropy will remain real except in the later case $\cosh (v / 2) \rightarrow \cos (v / 2)$. The entropy then has an oscillating part. In the locality limit $(\lambda \rightarrow 0): r_{-}^{2} \rightarrow 0, r_{+}^{2} \rightarrow m^{2}$ one obtains the case of the massive local scalar field theory. The other interesting limit is the massless limit $m \rightarrow 0$ : $r_{-}^{2} \rightarrow-i \lambda, \quad r_{+}^{2} \rightarrow i \lambda, \quad v$ becomes imaginary and $u \rightarrow$ $\sqrt{H^{4}+\lambda^{2}}$. In the massless limit the entropy acquires an oscillating part. This oscillation is entirely due to the presence of nonlocality. Also, as the end result is analytic in $m$ and $\lambda$ therefore the change of parameter $\lambda \rightarrow i \lambda$ remains well defined.

\section{Generic odd dimension}

In generic odd dimensions one can compute entanglement and Renyi entropy using the heat kernel given in
Eq. (50). This can then be plugged in Eq. (49) to obtain the heat kernel for the nonlocal theory considered here and consequently the effective action of the nonlocal theory following Eq. (48). These are then utilized in Eq. (46) which immediately gives the entropy. The whole complication resides in the computation of $F(n)$ in generic odd dimensions.

On applying a derivative with respect to $\mu$ on $K_{0}$ it is seen that it follows a recursive relation, which is true for any dimension. This recursion in $K_{0}$ also implies a recursion in $K_{1}$ given in Eq. (49).

$$
K_{1}^{d+2}(\mu, s)=-\frac{e^{-d H^{2} s} H}{2 \pi \sinh (H \mu)} \frac{\partial}{\partial \mu} K_{1}^{d}(\mu, s) .
$$

This recursive relation is useful in obtaining some of the terms of the entropy in generic dimensions. In general dimensions, we can define the following quantity:

$$
\begin{aligned}
I(d, s)= & \int_{0}^{\infty} \mathrm{d} \mu \sinh (H \mu)\left[1+\frac{\sinh ^{2}(H \mu / 2)}{\sin ^{2}(\omega / 2)}\right]^{(d-3) / 2} \\
& \times K_{1}^{d}(\mu ; s) .
\end{aligned}
$$

Using the recursive property of $K_{1}^{d}(\mu, s)$ stated in Eq. (55), it is noticed that $I(d, s)$ satisfies a corresponding recursive relation which is given by

$$
\begin{aligned}
I(d, s)= & \frac{H}{2 \pi} e^{-(d-2) H^{2} s} \\
& \times\left[K_{1}^{d-2}(0 ; s)+\frac{(d-3) H}{4 \sin ^{2}(\omega / 2)} I(d-2, s)\right] .
\end{aligned}
$$

By making use of such relations it is possible to compute $I(d, s)$ recursively. In particular, in spacetime with odd $d$ it contains only a finite number of terms in $I(d, s)$. Such a recursive relation offers an efficient algorithm in computing $I(d, s)$. In Appendix A we write $I(d, s)$ for some of the odd dimensions.

Once we have computed $I(d, s)$ then the next integration is performed over $\omega$ which is a contour integral. This involves computing residues. In Appendix A we write the expression for the result of the contour integration for some of the first few dimensions. Finally, one needs to perform the integration over $s$ whose limit ranges from $\delta^{2}$ and $\infty$. This integration reveals a Laurent series structure for $F(n)$ as the $s$ integration will have UV divergences.

$$
F(n)=\sum_{k=-(d-2)}^{\infty} F_{k}(n) \delta^{k},
$$


where $F_{k}(n)$ depend of the various parameters of the theory. The coefficient of negative powers of $\delta$ are the UV divergent pieces. The leading divergence comes entirely from the local physics and does not depend either on mass or nonlocality strength $\lambda$. Subleading divergences, however, depend on mass and nonlocality.
In Appendix A we have written $F_{k}(n)$ corresponding to UV divergence for some odd dimensions. In general their expression can be computed iteratively. The coefficient $F_{0}(n)$ is universal and $\mathrm{UV}$ finite. This contributes a universal piece to the entropy. For some of the odd dimensions it is given by

$$
\begin{aligned}
& F_{0}^{3}(n)=-\frac{i H\left(n^{2}-1\right) \sqrt{2}}{3 n}\left[\sqrt{2 H^{2}-\sqrt{m^{4}-4 \lambda^{2}}+m^{2}}\right. \\
& \left.+\sqrt{2 H^{2}+\sqrt{m^{4}-4 \lambda^{2}}+m^{2}}-\sqrt{2} H\right] \\
& F_{0}^{5}(n)=-\frac{i H\left(n^{2}-1\right)}{180 \pi n^{3}}\left[\left(116 H^{3} n^{2}-4 H^{3}+\sqrt{2} H^{2} \sqrt{8 H^{2}-\sqrt{m^{4}-4 \lambda^{2}}+m^{2}}\right.\right. \\
& +\sqrt{2} H^{2} \sqrt{8 H^{2}+\sqrt{m^{4}-4 \lambda^{2}}+m^{2}}-29 \sqrt{2} H^{2} n^{2} \sqrt{8 H^{2}-\sqrt{m^{4}-4 \lambda^{2}}+m^{2}} \\
& -29 \sqrt{2} H^{2} n^{2} \sqrt{8 H^{2}+\sqrt{m^{4}-4 \lambda^{2}}+m^{2}}-5 \sqrt{2} m^{2} n^{2} \sqrt{8 H^{2}-\sqrt{m^{4}-4 \lambda^{2}}+m^{2}} \\
& +5 \sqrt{2} n^{2} \sqrt{m^{4}-4 \lambda^{2}} \sqrt{8 H^{2}-\sqrt{m^{4}-4 \lambda^{2}}+m^{2}}-5 \sqrt{2} m^{2} n^{2} \sqrt{8 H^{2}+\sqrt{m^{4}-4 \lambda^{2}}+m^{2}} \\
& -5 \sqrt{2} n^{2} \sqrt{m^{4}-4 \lambda^{2}} \sqrt{8 H^{2}+\sqrt{m^{4}-4 \lambda^{2}}+m^{2}} \text {. }
\end{aligned}
$$

The interesting observation that should be made is that if we make a series of transformations:

$$
m \rightarrow \gamma m_{*}, \quad H \rightarrow \gamma H_{*}, \quad \lambda \rightarrow \gamma^{2} \lambda_{*} .
$$

(where $m_{*}, H_{*}$, and $\lambda_{*}$ are dimensionless parameters) then in odd dimensions the divergent contributions to the entropy are always odd in $\gamma$ while the universal finite term is always even in $\gamma$. This simple observation is very powerful in isolating the universal part of the entropy. In odd dimensional $d$ the universal finite piece is always a coefficient of $\gamma^{d-1}$. In the following we will be interested in determining this universal piece. We will obtain this universal piece using the Green's function.

\section{Universal finite piece from the Green's function}

In this subsection we will derive an expression for the universal piece of the entropy by making use of the Green's function. It should be observed that in odd dimensions the universal piece is the UV-finite part which arises for $\delta=0$. Also, after scaling (61) in odd dimensions the divergent part is odd while the universal part even. As a result the quantity $\left(S_{\gamma}+S_{-\gamma}\right) / 2$ being even in $\gamma$ will directly give the universal piece due to the cancellation of the all the UV divergent terms. In the following we will be interested in computing this quantity. This quantity can actually be written as $\frac{1}{2} \int_{0}^{\gamma} \mathrm{d} \gamma^{\prime} \frac{\partial S}{\partial \gamma^{\prime}}-\frac{1}{2} \int_{-\gamma}^{0} \mathrm{~d} \gamma^{\prime} \frac{\partial S}{\partial \gamma^{\prime}}=\frac{S(\gamma)+S(-\gamma)}{2}-S(0)$.

The quantity $S(0)$ is the entropy computed for $\gamma=0$, which results in $S(0)=0$. This immediately brings the focus on the computation of $\partial S / \partial \gamma$, which can be obtained from the Green's function of the theory. The effective action of the theory in terms of the heat kernel is written in Eq. (48). In this, if we do the scaling of parameters as indicated in Eq. (61), then the effective action is given by

$$
\begin{aligned}
W_{1}= & \lim _{x \rightarrow x^{\prime}} \int \mathrm{d} x \sqrt{g} \int_{0}^{\infty}\left(-\frac{\mathrm{d} s}{s}\right)\left(e^{-s \gamma^{2} r_{-}^{* 2}}+e^{-s \gamma^{2} r_{+}^{* 2}}-1\right) \\
& \times \operatorname{Tr} K_{0}\left(x, x^{\prime} ; s\right)
\end{aligned}
$$

where $K_{0}\left(x, x^{\prime} ; s\right)$ is the heat kernel for the $\square$ operator as before. On taking the derivative with respect to $\gamma$ we get

$$
\begin{aligned}
\frac{\partial W_{1}}{\partial \gamma}= & 2 \gamma \lim _{x \rightarrow x^{\prime}} \int \mathrm{d} x \sqrt{g} \int_{0}^{\infty} \mathrm{d} s\left(e^{-s \gamma^{2} r_{-}^{* 2}}+e^{-s \gamma^{2} r_{+}^{* 2}}\right) \\
& \times \operatorname{Tr} K_{0}\left(x, x^{\prime} ; s\right) .
\end{aligned}
$$

From this one can quickly recognize the form of the Green's function appearing in terms of the heat kernel, which is the standard definition of the Green's function in terms of the heat kernel [41]. 


$$
\begin{aligned}
& G_{+}\left(x, x^{\prime}\right)=\int_{0}^{\infty} \mathrm{d} s e^{-s r_{+}^{2}} K_{0}\left(x, x^{\prime} ; s\right), \\
& G_{-}\left(x, x^{\prime}\right)=\int_{0}^{\infty} \mathrm{d} s e^{-s r_{-}^{2}} K_{0}\left(x, x^{\prime} ; s\right) .
\end{aligned}
$$

The Green's functions $G_{-}$and $G_{+}$have also been previously obtained by solving the hypergeometric differential equation. They satisfy Eq. (20) and have solutions given in Eq. (23) with $q_{-}$and $q_{+}$given in Eq. (25). These are known exactly in AdS background as have been shown in Sec. II B in closed form and are also well known from literature [23-26]. In the following we will be using these to compute the expression for $\partial S / \partial \gamma$.

The Green's function $G_{-}$and $G_{+}$can be suitably generalized to obtain on the $n$ copies of AdS, the following Sommerfeld formula which leads to the following expression for the Green's function on the $n$ manifold $\mathcal{M}^{n}$ :

$$
\left(G_{n}^{ \pm}-G_{1}^{ \pm}\right)\left(\phi, \phi^{\prime}\right)=\frac{i}{4 \pi n} \int_{\Gamma} \mathrm{d} \omega \cot \left(\frac{\omega}{2 n}\right) G_{1}^{ \pm}\left(\phi-\phi^{\prime}+\omega\right) .
$$

This will give a corresponding effective action on $\mathcal{M}^{n}$ following Eq. (64).

$$
\begin{aligned}
\frac{\partial W_{n}}{\partial \gamma}= & 2 \gamma \operatorname{Tr}\left[G_{n}^{+}\left(x, x ; m^{2}\right)+G_{n}^{-}\left(x, x ; m^{2}\right)\right] \\
= & 2 \gamma H^{3-d} \int_{0}^{2 \pi n / H} \mathrm{~d} \tau_{E} \times \int_{H^{d-2}} \mathrm{~d} V_{d-2} \\
& \times \int_{0}^{\infty} \mathrm{d} \rho \rho\left(1+H^{2} \rho^{2}\right)^{\frac{d-3}{2}} \\
& \times\left[G_{n}^{+}\left(x, x ; m^{2}\right)+G_{n}^{-}\left(x, x ; m^{2}\right)\right],
\end{aligned}
$$

where $G_{n}^{ \pm}\left(x, x^{\prime}\right)$ is obtained in Sec. II B. This allows us to translate the heat kernel and the Green's function of the scalar in EAdS to the heat kernel and Green's function on the metric equation (39), respectively. These will be used in the computation of entanglement entropy (and Renyi entropy). In this case we have

$$
\begin{aligned}
& \frac{\partial S_{\mathrm{EE}}}{\partial \gamma}=\lim _{n \rightarrow 1}-\frac{i}{2} \frac{\mathcal{A}(\Sigma)}{H^{d-2}}\left(n \partial_{n}-1\right) F^{G}(n), \\
& \frac{\partial S_{\mathrm{Renyi}}}{\partial \gamma}=\lim _{n \rightarrow 1}-\frac{i}{2(n-1)} \frac{\mathcal{A}(\Sigma)}{H^{d-2}} F^{G}(n),
\end{aligned}
$$

where

$$
\begin{aligned}
F^{G}(n)= & 2 \gamma \int_{0}^{\infty} \mathrm{d} \rho \rho\left(1+H^{2} \rho^{2}\right)^{\frac{d-3}{2}} \int_{\Gamma} \mathrm{d} \omega \cot \left(\frac{\omega}{2 n}\right) \\
& \times\left[G_{1}^{+}(\mu)+G_{1}^{-}(\mu)\right],
\end{aligned}
$$

$\cosh ^{2}\left(\frac{H \mu}{2}\right)=z=1+(H \rho)^{2} \sin ^{2}(\omega / 2)$ (as before) and $G_{1}^{ \pm}(\mu)$ is the EAdS Green's function computed previously using the differential equation. A change of variable which leads $\mathrm{d} z=2 H^{2} \rho \sin ^{2}(\omega / 2) \mathrm{d} \rho$ with the parameter $z$ range $1 \leq z \leq \infty$. Then we choose to first perform the integration over $\omega$ and then over $z$. This interchange of the order of integration leads to

$$
\begin{aligned}
F^{G}(n)= & \frac{\gamma}{H^{2}} \int_{1}^{\infty} \mathrm{d} z\left[G_{1}^{+}(z)+G_{1}^{-}(z)\right] \int_{\Gamma} \mathrm{d} \omega \frac{\cot (\omega / 2 n)}{\sin ^{2}(\omega / 2)} \\
& \times\left[1+\frac{z-1}{\sin ^{2}(\omega / 2)}\right]^{(d-3) / 2} .
\end{aligned}
$$

In generic odd dimensions $d=2 j+1$ (where $j \in \mathbb{I}$ ) one has the following polynomial expansion with finite number of terms in expansion:

$$
\left[1+\frac{z-1}{\sin ^{2}(\omega / 2)}\right]^{j-1}=\sum_{k=0}^{j-1} \frac{(j-1) !}{k !(j-k-1) !}\left(\frac{z-1}{\sin ^{2}(\omega / 2)}\right)^{k}
$$

In even dimensions this binomial expansion has an infinite number of terms in series. In odd dimensional AdS, using this expansion one can perform the $\omega$ integration, which leads to the following expression for $F^{G}(n)=$ $\left(\gamma / H^{2}\right) \sum_{k} F_{k}^{G}$ with $F_{k}^{G}$,s being the function of $n$ given by

$$
F_{k}^{G}=\frac{\Gamma(d-2) C_{k}(n)}{\Gamma(k) \Gamma(d-2+k)} \int_{1}^{\infty} \mathrm{d} z(z-1)^{k}\left[G_{1}^{+}(z)+G_{1}^{-}(z)\right],
$$

where the functions $C_{k}(n)$ are obtained during the process of computing the contour integration.

$$
C_{k}(n)=\int_{\Gamma} \mathrm{d} \omega \cot \left(\frac{\omega}{2 n}\right)\left(\sin \left(\frac{\omega}{2}\right)\right)^{-(2 k+2)} .
$$

The functions $C_{k}(n)$ are coefficients containing information of the background involving conical singularity $[33,34]$ and are rational functions in $n$. In Appendix B we write some of the first few $C_{k}$ 's. The integration over $z$ as given in Eq. (73) can be performed on Mathematica giving a closed form expression in terms of hypergeometric functions. This is given by 


$$
\begin{aligned}
\int_{1}^{\infty}(z-1)^{k}\left[G_{1}^{+}(z)+G_{1}^{-}(z)\right]= & \Gamma(k+1)\left[\frac{q_{-} \Gamma\left(a_{-}-k-1\right)}{\Gamma\left(a_{-}\right)}\right. \\
& \times{ }_{2} F_{1}\left(a_{-}-k-1, a_{-}-c_{-}+1, a_{-}-b_{-}+1,1\right)+\frac{q_{+} \Gamma\left(a_{+}-k-1\right)}{\Gamma\left(a_{+}\right)} \\
& \left.\times{ }_{2} F_{1}\left(a_{+}-k-1, a_{+}-c_{+}+1, a_{+}-b_{+}+1,1\right)\right],
\end{aligned}
$$

where we have used the structure of the Green's functions $G_{-}$and $G_{+}$on EAdS obtained previously, with $q_{-}$and $q_{+}$given in Eq. (25), respectively. The hypergeometric function has a singularity at argument $z=1$, but for now we do not worry about it. At this point one can combine Eqs. (73)-(75) to obtain an expression for $F^{G}(n)$ given in Eq. (71). This is the main ingredient that is required for the computation of the entanglement or Renyi entropy. The function $F^{G}(n)$ is given by

$$
\begin{aligned}
F^{G}(n)= & \frac{\gamma}{H^{2}} \sum_{k=0}^{j-1} \frac{\Gamma(d-2) C_{k}(n)}{\Gamma(k) \Gamma(d-2+k)} \Gamma(k+1) \\
& \times\left[\frac{q-\Gamma\left(a_{-}-k-1\right)}{\Gamma\left(a_{-}\right)}{ }_{2} F_{1}\left(a_{-}-k-1, a_{-}-c_{-}+1, a_{-}-b_{-}+1,1\right)\right. \\
& \left.+\frac{q_{+} \Gamma\left(a_{+}-k-1\right)}{\Gamma\left(a_{+}\right)}{ }_{2} F_{1}\left(a_{+}-k-1, a_{+}-c_{+}+1, a_{+}-b_{+}+1,1\right)\right]
\end{aligned}
$$

At this point one can do the scaling of parameters using Eq. (61). From the resulting expression we compute the universal finite part of entropy following Eqs. (68) and (69) by integrating with respect to $\gamma$ and using Eq. (62). At this point we notice that the $n$ derivate and the $n$ limit act directly on the functions $C_{k}(n)$. This series has a finite number of terms and offers a closed form expression. In the computation of entropy this is the universal piece.

\section{CONCLUSIONS}

In this paper we have considered a nonlocal scalar field theory and investigated entanglement entropy in this theory in odd AdS spacetime.

First, the Green's function of the theory in flat and AdS spacetime was computed and analyzed. Using the trick of partial decomposition we wrote the propagator of nonlocal theory as a linear sum propagator of two local theories. The Green's function so computed is well behaved in ultraviolet (short distances) and infrared (long distances). The massless limit of the nonlocal propagator in AdS is well defined.

We then calculated the entanglement entropy in this theory with the purpose to investigate whether nonlocality can play a significant role in affecting long-distance entanglement. We used the replica trick. For this we first computed the expression for the effective action of the nonlocal theory. This was then written in the language of the heat kernel on the AdS. Using the Sommerfeld formula we then computed the heat kernel and subsequently the effective action on the $n$ copies of the manifold $\mathcal{M}^{n}$. This allowed us to compute the entanglement and Renyi entropy via replica trick. We computed the entropy in odd spacetime dimensions. We found that the area law is followed even for nonlocal theory. The leading term of the entropy is independent of any of the parameters of the theory. The subleading terms depend on mass and nonlocality strength. This is expected in the sense that the leading term arises because of the ultralocal physics and hence does not contain any dependence on mass or nonlocality.

In odd dimensions the entropy is seen to have a universal part which is UV finite. We computed the form of this universal part of the entropy in all odd dimensions using the Green's functions, which offer a quick and elegant way to compute universal contributions to entropy. This UV-finite piece, unlike the leading term, has more structure and incorporates the effect from nonlocality. In $\mathrm{AdS}_{3}$, this universal part is seen to be oscillating in massless theories. This oscillation is entirely due to the presence of nonlocality in the theory, which does not happen in case of local theories [6], and can be seen as a novelty due to the presence of nonlocality. The oscillation can be understood physically by expressing the nonlocal theory as a local one, where one can notice the presence of a tachyon or a complex pole. This is only indicating that the nonlocal theory is an effective one in infrared regimes and cannot become a fundamental local theory.

\section{ACKNOWLEDGMENTS}

N. K. is supported by the SERB National Postdoctoral Fellowship. G. N. is supported by the "Zhuoyue" Fellowship (ZYBH2018-03). 


\section{APPENDIX A: $I(d, s)$ IN ODD $d$}

Here we write the expression for $I(d, s)$ for some of the odd dimensions. $I(d, s)$ is given in Eq. (56) and follows a recursive relation mentioned in Eq. (57).

$$
\begin{gathered}
I(3, s)=\frac{H e^{-H^{2} s}\left[2 e^{-s m^{2} / 2} \cosh \left(\frac{1}{2} s \sqrt{m^{4}-4 \lambda^{2}}\right)-1\right]}{4 \pi^{3 / 2} \sqrt{s}}, \\
I(5, s)=-\frac{H e^{-4 H^{2} s}\left(H^{2} s \csc ^{2}\left(\frac{\omega}{2}\right)+1\right)\left[1-2 e^{-m^{2} s / 2} \cosh \left(\frac{1}{2} s \sqrt{m^{4}-4 \lambda^{2}}\right)\right]}{16 \pi^{5 / 2} s^{3 / 2}}, \\
I(7, s)=-\frac{H e^{-9 H^{2} s}}{192 \pi^{7 / 2} s^{5 / 2}}\left[1-2 e^{-m^{2} s / 2} \cosh \left(\frac{1}{2} s \sqrt{m^{4}-4 \lambda^{2}}\right)\right] \\
\times\left[\frac{6 H^{4} s^{2}}{\sin ^{4}(\omega / 2)}+\frac{6 H^{2} s}{\sin ^{2}(\omega / 2)}+2 H^{2} s+3\right] .
\end{gathered}
$$

Once the $I(d, s)$ are known, one can compute the contour integration over $\omega$. The integral that we are interested in performing is

$$
J(d, n, s)=\frac{\text { in }}{\left(n^{2}-1\right) H} \int_{\Gamma} \mathrm{d} \omega \frac{\cot (\omega / 2 n)}{\sin ^{2}(\omega / 2)} I(d, s) .
$$

For some of the first few odd dimensions they are given by

$$
\begin{gathered}
J(3, n, s)=\frac{e^{-H^{2} s}\left[1-2 e^{-m^{2} s / 2} \cosh \left(\frac{1}{2} s \sqrt{m^{4}-4 \lambda^{2}}\right)\right]}{3 \sqrt{s \pi}}, \\
J(5, n, s)=\frac{e^{-4 H^{2} s}\left[1-2 e^{-m^{2} s / 2} \cosh \left(\frac{1}{2} s \sqrt{m^{4}-4 \lambda^{2}}\right)\right]\left[n^{2}\left(11 H^{2} s+15\right)+H^{2} s\right]}{180 n^{2}(s \pi)^{3 / 2}}, \\
J(7, n, s)= \\
\quad \frac{e^{-9 H^{2} s}\left[1-2 e^{-m^{2} s / 2} \cosh \left(\frac{1}{2} s \sqrt{m^{4}-4 \lambda^{2}}\right)\right]}{15120 n^{4}(s \pi)^{5 / 2}} \\
\times\left[2 H^{4}\left(191 n^{4}+23 n^{2}+2\right) s^{2}+42 H^{2} n^{2}\left(16 n^{2}+1\right) s+315 n^{4}\right] .
\end{gathered}
$$

From this one can compute $F(n)$ following Eq. (46) and the various divergent pieces of $F(n)$. Below we will mention divergent pieces of $F(n)$ for some odd dimensions.

$$
\begin{gathered}
F_{-1}^{3}=\frac{2 i H\left(n^{2}-1\right)}{3 \sqrt{\pi} n}, \\
F_{-3}^{5}=\frac{i H\left(n^{2}-1\right)}{18 \pi^{3 / 2} n}, \quad F_{-1}^{5}=-\frac{i H\left(n^{2}-1\right)\left(49 H^{2} n^{2}-H^{2}+15 m^{2} n^{2}\right)}{90 \pi^{3 / 2} n^{3}}, \\
F_{-5}^{7}=\frac{i H\left(n^{2}-1\right)}{120 \pi^{5 / 2} n}, \quad F_{-3}^{7}=-\frac{i H\left(n^{2}-1\right)\left(103 H^{2} n^{2}-2 H^{2}+15 m^{2} n^{2}\right)}{1080 \pi^{5 / 2} n^{3}}, \\
F_{-1}^{7}=\frac{i H\left(n^{2}-1\right)}{15120 \pi^{5 / 2} n^{5}}\left(14183 H^{4} n^{4}-664 H^{4} n^{2}+8 H^{4}+4326 H^{2} m^{2} n^{4}-84 H^{2} m^{2} n^{2}\right. \\
\left.+315 m^{4} n^{4}-630 \lambda^{2} n^{4}\right) .
\end{gathered}
$$

If we do scaling $m \rightarrow \gamma m_{*}, H \rightarrow \gamma H_{*}$, and $\lambda \rightarrow \gamma^{2} \lambda_{*}$ (where $m_{*}, H_{*}$, and $\lambda_{*}$ are dimensionless parameters) then it will be seen that all these are odd in $\gamma$. 


\section{APPENDIX B: $C_{k}(n)$}

Here we just mention some of the first few $C_{k}(n)$ following the residue theorem for the evaluation of contour integral.

$$
\begin{gathered}
C_{0}(n)=\frac{4 i \pi\left(n^{2}-1\right)}{3 n}, \\
C_{1}(n)=\frac{4 i \pi\left(n^{2}-1\right)\left(11 n^{2}+1\right)}{45 n^{3}}, \\
C_{2}(n)=\frac{4 i \pi\left(n^{2}-1\right)\left(191 n^{4}+23 n^{2}+2\right)}{945 n^{5}},
\end{gathered}
$$

$$
C_{3}(n)=\frac{4 i \pi\left(n^{2}-1\right)\left(11 n^{2}+1\right)\left(227 n^{4}+10 n^{2}+3\right)}{14175 n^{7}},
$$

$$
\begin{aligned}
& C_{4}(n) \\
& =\frac{4 i \pi\left(n^{2}-1\right)\left(14797 n^{8}+2125 n^{6}+321 n^{4}+35 n^{2}+2\right)}{93555 n^{9}} \\
& C_{5}(n)=\frac{4 i \pi\left(n^{2}-1\right)}{638512875 n^{11}}\left(92427157 n^{10}+13803157 n^{8}\right. \\
& \left.\quad+2295661 n^{6}+307961 n^{4}+28682 n^{2}+1382\right) .
\end{aligned}
$$

[1] L. Bombelli, R. K. Koul, J. Lee, and R. D. Sorkin, Phys. Rev. D 34, 373 (1986).

[2] M. Srednicki, Phys. Rev. Lett. 71, 666 (1993).

[3] H. Casini and M. Huerta, J. Phys. A 42, 504007 (2009).

[4] S. Ryu and T. Takayanagi, J. High Energy Phys. 08 (2006) 045.

[5] T. Faulkner, A. Lewkowycz, and J. Maldacena, J. High Energy Phys. 11 (2013) 074.

[6] S. Sugishita, J. High Energy Phys. 09 (2016) 128.

[7] N. Shiba and T. Takayanagi, J. High Energy Phys. 02 (2014) 033.

[8] J. L. Karczmarek and C. Rabideau, J. High Energy Phys. 10 (2013) 078.

[9] W. Fischler, A. Kundu, and S. Kundu, J. High Energy Phys. 01 (2014) 137.

[10] D. W. Pang, Phys. Rev. D 89, 126005 (2014).

[11] D. Nesterov and S. N. Solodukhin, Nucl. Phys. B842, 141 (2011).

[12] D. Nesterov and S. N. Solodukhin, J. High Energy Phys. 09 (2010) 041.

[13] S. N. Solodukhin, Living Rev. Relativity 14, 8 (2011).

[14] L. Modesto, Phys. Rev. D 86, 044005 (2012).

[15] L. Modesto and L. Rachwał, Int. J. Mod. Phys. D 26, 1730020 (2017).

[16] M. Maggiore, Fundam. Theor. Phys. 187, 221 (2017).

[17] G. Narain and T. Li, Phys. Rev. D 97, 083523 (2018).

[18] G. Narain and T. Li, Universe 4, 82 (2018).

[19] N. Kajuri, Phys. Rev. D 95, 101701 (2017).

[20] N. Kajuri and D. Kothawala, Phys. Lett. B 791, 319 (2019).

[21] N. Kajuri and G. Narain, arXiv:1812.00946.
[22] S. J. Avis, C. J. Isham, and D. Storey, Phys. Rev. D 18, 3565 (1978).

[23] B. Allen and T. Jacobson, Commun. Math. Phys. 103, 669 (1986).

[24] C. P. Burgess and C. A. Lutken, Phys. Lett. 153B, 137 (1985).

[25] M. M. Caldarelli, Nucl. Phys. B549, 499 (1999).

[26] S. S. Gubser and I. Mitra, Phys. Rev. D 67, 064018 (2003).

[27] G. Narain and N. Kajuri, Phys. Lett. B 791, 143 (2019).

[28] C. G. Callan, Jr. and F. Wilczek, Phys. Lett. B 333, 55 (1994).

[29] C. Holzhey, F. Larsen, and F. Wilczek, Nucl. Phys. B424, 443 (1994).

[30] P. Calabrese and J. L. Cardy, J. Stat. Mech. (2004) P06002.

[31] P. Calabrese and J. Cardy, J. Phys. A 42, 504005 (2009).

[32] J. S. Dowker, J. Phys. A 10, 115 (1977).

[33] J. S. Dowker, Phys. Rev. D 36, 3095 (1987).

[34] D. V. Fursaev, Phys. Lett. B 334, 53 (1994).

[35] A. Sommerfeld, Proc. London Math. Soc. s1-28, 395 (1896).

[36] H. Casini, M. Huerta, and R. C. Myers, J. High Energy Phys. 05 (2011) 036.

[37] L. Y. Hung, R. C. Myers, and M. Smolkin, J. High Energy Phys. 10 (2014) 178.

[38] R. Camporesi, Phys. Rep. 196, 1 (1990).

[39] S. Giombi, A. Maloney, and X. Yin, J. High Energy Phys. 08 (2008) 007.

[40] J. R. David, M. R. Gaberdiel, and R. Gopakumar, J. High Energy Phys. 04 (2010) 125.

[41] B. S. DeWitt, Conf. Proc. C630701, 585 (1964); Les Houches Lect. Notes 13, 585 (1964). 